\title{
Is the Quantification of Mental Stress Possible?
}

\author{
Koji Hori*, Kimiko Konishi and Michiho Sodenaga \\ Department of Neuropsychiatry, St Marianna University School of Medicine, Japan
}

Submission: March 09, 2017; Published: March 20, 2017

*Corresponding author: Koji Hori, Department of Neuropsychiatry, St Marianna University, School of Medicine, Japan, Tel: +81-44-977-8111; Fax: +81-44-977-8111; Email: kojihori@marianna-u.ac.jp

\section{Opinion}

We are studying the relationships among inflammation, anticholinergic activity (AA), and clinical symptoms including cognitive function and behavioral and psychological symptoms of dementia in neuropsychiatric disorders and neurocognitive disorders such as Alzheimer's disease (AD) and Lewy body disease (LBD).

In this context, we proposed the hypothesis of endogenous appearance of $\mathrm{AA}$ in $\mathrm{AD}$ and $\mathrm{LBD}[1,2]$. In $\mathrm{AD}$, when down regulation of acetylcholine (ACh) reaches the critical level, the inflammatory system is up regulated and cytokines with AA appears endogenously [1]. In case of LBD, although down regulation of Ach dosen't reach the critical level, the other AA insert such as mental stress (hyper activation of HPA axis) is added and inflammatory system is also up regulated and cytokines with AA appears endogenously [2]. However, even in the later cases the down regulation of ACh. is essential for the appearance of AA. If the cholinergic system is intact, the up regulation of this system is possible, which can compensate this up regulated HPA axis and doesn't allow to up regulate the inflammatory system [3]. We speculate that this mechanism is "the overcome the mental stress". In fact, when the stress is added, sickness behaviors occur [4]. We also speculate this sickness behaviors are related with the up regulation of $\mathrm{AC}$ hand that these sickness behaviors appear as the compensatory mechanisms of mental stress. In in this context persons with deteriorated ACh and system mental stress should be avoided. However, overcome of the mental stress is needed in order to development of brain nervous system in healthy persons those their cholinergic systems are intact [5,6]. Moreover, not only pharmacological treatment but also non pharmacological treatment is effective the ameliorations of mental stresses [7]. These two conditions (the over activated inflammatory state and hypoactivity of ACh as compensatory mechanism) are similar with each other.
Can we distinguish between this right situation and wrong situation? In "right situation", the cholinergic system is up regulated in order not to upregulate the inflammatory system for coping of mental stress. On the contrary to this, in "wrong situation", the cholinergic system is not enough up regulated in order not to up regulate the inflammatory system for coping of mental stress. Of course, in this "wrong situation", the inflammatory system is over activated. From these, we consider that some inflammatory markers can distinguish between these two situations. Among these, we propose the serum anticholinergic activity (SAA). SAA is peripheral marker of anticholinergic burden, which is related with inflammation. Therefore, we consider SAA as an inflammatory marker in peripheral system. Although as peripherally evaluated, SAA is reflect the inflammatory system in the central nervous system because inflammation in peripheral tissue and that in central nervous system are connected. When the mental stress is added and SAA is negative, the person is in "right situation", we should conduct the nonpharmacological therapy in order to offer the enrich environment. When the mental stress is added and SAA is positive, the person is in "wrong situation", we should prescribe the cholinesterase inhibitor as pharmacological therapy in order to abolish AA.

In these point of view, it is important to resume the quantification of SAA. Because of radiation control, the evaluation of SAA is now stopped. We are now trying to resume the quantification of SAA.

\section{Conflict of Interest}

Koji Hori received lecture fees from Eisai Co. Ltd., Pfizer Japan Inc., Novartis Pharma KK, Daiichi Sankyo Inc., Ono Pharmaceutical Co. Ltd., Janssen Pharmaceutical KK, Yoshitomi Yakuhin Co. Meiji Seika Pharma Co. Ltd., and Mitsubishi Tanabe Pharma Co.However, the sponsors had no role in study design, 
data collection and analysis including our before presented articles, decision to publish, or preparation of this manuscript.

\section{Disclosure Statement}

Koji Hori funding from Ito Memorial Foundation. However, these funding are not used for this article.

\section{Author Contributions}

Koji Hori coordinated the study about the inflammatory markers in neuropsychiatric disorders. Kimiko Konishiis now trying to measure serum anticholinergic activity as the biological marker of inflammation, and MichihoSodenaga gave idea about treatment option against inflammation and anticholinergic activity in psychiatric disorders. Kimiko Konishiand MichihoSodenagaalso checked the manuscript.

\section{Acknowledgment}

Dr. Chiaki Hashimoto (St. Mariana University School of Medicine, Department of Neuropsychiatry, Kawasaki, Japan), Dr. Mioto Maedomari(St. Mariana University Yokohama Western Hospital, Department of Neuropsychiatry, Yokohama, Japan), Dr. Ouga Sasaki (St. Mariana University School of Medicine, Department of Neuropsychiatry, Kawasaki, Japan), Dr. ItsukuSuziki (Kawasaki Municipal Tama Hospital, Department of Neuropsychiatry, Kawasaki, Japan) and Dr. Hiroyuki Kamatani (Kawasaki Memorial Hospital Department of Psychiatry, Kawasaki, Japan) are other member in study group of geropsychiatry inSt. Mariana
University School of Medicineforstudies in geropsychiatric patients.

\section{References}

1. Hori K, Konishi K, Hosoi M, Tomioka H, Tani M, et al. (2016) Mini Review: Anticholinergic activity as a behavioral pathology of Lewy Body disease and proposal of the concept of anticholinergic spectrum disorders". Parkinsons Dis 2016: 5380202.

2. Konishi K, Hori K, Tani M, Tomioka H, Kitajima Y, Akashi N, et al. (2015) Hypothesis of endogenous anticholinergic activity in Alzheimer's disease. Neurodegener Dis 15(3): 149-156.

3. Hori K, Hachisu M, Tomioka H, Konishi K (2015) Anticholinergic activity and Alzheimer's disease. Neurodegener Dis 15(3): 131-133.

4. Kirsten TB, Galvão MC, Reis-Silva TM, Queiroz-Hazarbassanov N, Bernardi MM (2015) Zinc prevents sickness behavior induced by lipopolysaccharides after a stress challenge in rats. PLoS One 10(3): e0120263.

5. Anacker C, Zunszain PA, Carvalho LA, Pariante CM (2011) The glucocorticoid receptor: pivot of depression and of antidepressant treatment? Psychoneuroendocrinology 36(3): 415-425.

6. Lucassen PJ, Oomen CA, Naninck EF, Fitzsimons CP, van Dam AM, et al. (2015) Regulation of Adult Neurogenesis and Plasticity by (Early) Stress, Glucocorticoids, and Inflammation. Cold Spring Harb Perspect Biol 7(9): a021303.

7. Bhagya V, Srikumar BN, Veena J, Shankaranarayana Rao BS (2016) Short-term exposure to enriched environment rescues chronic stressinduced impaired hippocampal synaptic plasticity, anxiety, and memory deficits. J Neurosci Res. 